\title{
Mobility in Wireless Networks
}

\author{
Christian Schindelhauer* \\ Heinz Nixdorf Institute, University of Paderborn, Germany \\ schindel@uni-paderborn.de
}

\begin{abstract}
This article surveys mobility patterns and mobility models for wirelss networks. Mobility patterns are classified into the following types: pedestrians, vehicles, aerial, dynamic medium, robot, and outer space motion. We present the characteristics of each and shortly mention the specific problems.

We shortly present the specifics of cellular networks, mobile ad hoc networks, and sensor networks regarding mobility. Then, we present the most important mobility models from the literature. At last we give a brief discussion about the state of research regarding mobility in wireless networks.
\end{abstract}

\section{Introduction}

Today, it is hard to imagine the difficulties to send information over large distances before the invention of radio communication. First, such devices were so large and heavy that they could not be carried around but had to be carried by vehicles. So, the history of mobile communication starts with radio devices on boats which emerged in the 1890s and helped ocean vessels to overcome insulation for navigation and emergency situations. It took some time until mobile radio transceiver could be used on non-marine vehicles. Such technology was available in the 1930s when a radio transceiver could be operated on a bicycle and a radio sender could be operated on an airplane. Both as show cases and not really for practical use. At the end of the 30s portable solutions were available in form of the famous "walky-talky", which could be carried by a single person. In the beginning of the 1940s a radio transceiver was available which could be held in a single hand: "The Handy-Talky", see Fig. 1. Needless to say that these communication devices played an important role in the second world war. With the upcoming of transistors, large scale integrated chip layout, and new battery technology allowed the size of radio devices to shrink unbelievably small. Today, one can buy fully equipped sensor nodes with radio transceiver and micro-controller in the size of a small coin (and within reasonable price), see Fig. 2. Furthermore, there is the vision of communication devices being so small called "smart dust".

\footnotetext{
* Supported by the DFG Sonderforschungsbereich 376: "Massive Parallelität: Algorithmen, Entwurfsmethoden, Anwendungen." and by the EU within the 6th Framework Programme under contract 001907 "Dynamically Evolving, Large Scale Information Systems" (DELIS).
}

J. Wiedermann et al. (Eds.): SOFSEM 2006, LNCS 3831, pp. 100-116 2006.

(C) Springer-Verlag Berlin Heidelberg 2006 


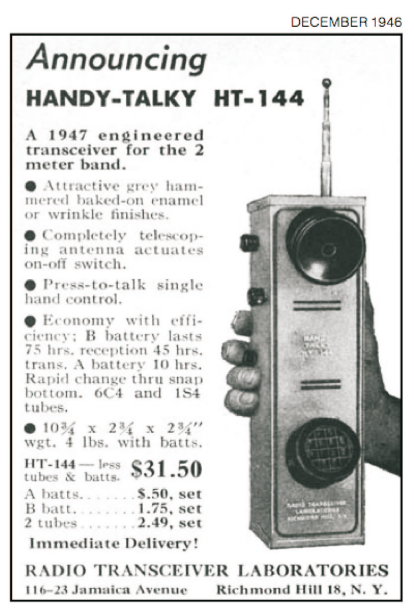

Fig. 1. The Handy-Talky

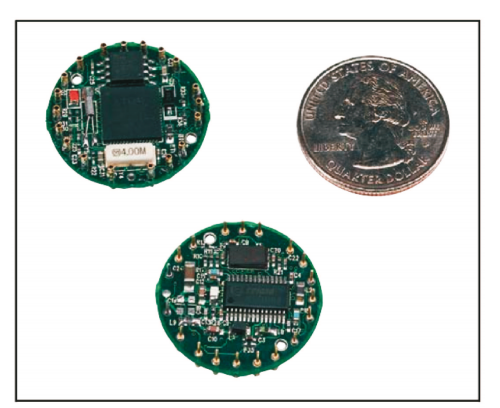

Fig. 2. The Mica2Dot from Crossbow

Also the underlying radio communication technology has changed much from the analogous broadcast signal (still surprisingly widely distributed) to digital multi-hop networks with time, frequency and code division multiplexing for the parallel use of the medium. Such packet oriented radio devices have been developed in the 1970s and filled the interior of a van, i.e. Vint Cerf's Stanford Research Institute (SRI) van. Then, the packet radio underwent a miniaturization process and packet radio has become the dominating radio technology, so far.

For two-way radio communication central radio stations are used which serve as relay station for transmitting the radio signals. Many of these central relay stations partition the radio landscape into cells. In such cellular networks the mobility of users (more or less) reduces to problems of handover from one radio station to a neighbored station. Networks without such centralized infrastructure are called mobile ad hoc networks. There, the impact of mobility is much higher, since everything is moving. One can easily imagine the possible negative impact of mobility on wireless networking. Recent results point out that mobility has also a positive impact.

This article surveys mobility and radio networks from a wide perspective. We refrain from going into the very details of mobility aspects and head for giving a broader picture. The goal of this survey is to endorse new approaches to mobility in wireless networks based on the current situation. For this, we discuss on mobility patterns, mobility models, algorithmic aspects and on mobile ad hoc networks. Very often the mobility models and mobility patterns are mixed up. However, one must carefully distinguish between them. Real mobility pattern can be obtained by tracking moving objects of reality, while mobility models try to generalize such patterns by forming a mathematical model.

We begin with a very short introduction of cellular networks, ad hoc networks, and wireless sensor networks. Then, we continue with an overview how and where mobility occurs and how it might affect wireless networks. After that we 
elaborate mobility models from literature. In the last section we present positive aspects of mobility on the wireless networks, research perspectives, and open research problems.

This is not the first survey on mobility of wireless networks. There is an excellent survey paper of Camp, Boleng and Davies [12] which presents and discusses mobility models for ad hoc networks. It is based on the more detailed $\mathrm{PhD}$ thesis of Vannessa A. Davies [17. A survey of random direction based models can be found in [41.

\section{Wireless Networks in a Nutshell}

The radio frequency spectrum is divided into several bands, starting as low as $30 \mathrm{kHz}$ for maritime communication and ranging up to $300 \mathrm{GHz}$. Low frequency radio waves easily pass through human bodies, walls, water, etc. Higher frequencies are absorbed and reflected by obstacles. There are numerous other facts to be told from physics. For some frequency bands the ionosphere reflects signals. The background noise level differs in various bands. Signal strength is influenced by obstruction, diffusion, reflection, multi-path fading, and the Doppler effect. A man-made problem is the interference of radio signals of multiple senders.

Furthermore, in theory the signal strength is fading with a power of two with respect to the distance, which is only true in empty space. In other environments the exponent is larger and can have values from 3-5. This implies that if one tries to send over a distance of $d$ transmission power has to chosen $\sim d^{2}$ in empty space. Many transmission models assume that the covered area by a radio signal can be modeled by a disc. However, practical measurements show that this is not at all the case.

\subsection{Cellular Networks}

Cellular networks are defined by static base stations which divide the fields into cells. All radio communication is between these base stations and the clients. Usually, each static base station forwards and receives packets to other base stations by another (hard-wired) network. Regarding movement of clients one is only interested in whether the node enters or leaves a cell. It is not interesting where the node is exactly located within a cell. In some cellular networks (like UMTS) the size of the cell changes with the number of nodes. Usually network cells overlap and so, there are areas where a client can choose among several base stations.

The main mobility problems and applications for cellular networks are Cellular Handoff: Provide a robust protocol that allows to move between cells without interrupting and disturbing communication; Location Service: Use the cell information and the power strength to locate a client within the network.

\subsection{Mobile Ad Hoc Networks}

A Mobile Ad Hoc Wireless Network (MANET) is a self-configuring network of mobile nodes. Nodes serve as routers and may move arbitrarily. There is no 
static infrastructure and the communication network must be able to adapt to changes because of movement and other dynamics. Most of the MANET protocols do not assume that position data is available. However, if such position data is available then efficient location based communication protocols are applicable (for a survey on such routing algorithms see 39]). The main problem in MANET is to find a multi-hop route between the source and the target of information. It is clear that if all the intermediate router nodes are moving that this type of network is very much affected by mobility. Especially if one takes into account that the transmitting range is rather restricted to a limited supply of energy (e.g. batteries). See [44] as an introduction to ad hoc networks.

The main mobility problems for a MANET are routing a message, multicasting a message, and upholding the network routing tables for these issues.

\subsection{Sensor Networks}

A sensor network is a wireless network connecting many, specially distributed sensor devices. These devices are equipped with sensors, such as for temperature, vibration, pressure, sound, or motion. Like in a mobile ad hoc network this information has to be communicated without a special infrastructure over a multi-hop path. Similar as for cellular networks there are specially equipped base stations (sometimes connected over an ad hoc network) to collect this information and control the network.

The main difference between a sensor network and a mobile ad hoc network is that a sensor network is data-driven. It is important to receive the temperature reading of an area not from a specific device. So, some of the sensors may be switched off for most of the time. Furthermore, these cheap and massively deployed sensors are equipped with the bare minimum of computing resources. Sometimes they have to work for some 10 years being solely powered by small coin cell batteries.

The main application of a sensor network is to read out the sensor reading of a particular area. At the moment mobile sensor networks are the exception (which we will discuss here). For surveys on sensor networks, see [14, 30, [46], [57].

\section{Mobility Patterns: How People and Things Move}

We now give a overview over realistic mobility patterns and classify them as follows: pedestrians, vehicles, aerial, dynamic medium, robot, and outer space motion. We present their characteristics and mention the specific radio problems.

\subsection{Pedestrians}

The oldest and most common way of mobility is to walk. Pedestrian mobility has the slowest velocity compared to other modern mobility patterns. Although low in speed, even in cellular networks walking patterns cause sincere trouble 
since typically people walk through places where obstacles obstruct the signal. It is often a matter of meters whether or not the access point is to be reached by a client (fast signal fading). In such a case clients need to be handed over rapidly to the next available access point.

So, pedestrian mobility describes the walking patterns of people or animals. Its main characteristics are the full use of the two-dimensional plane with occasional obstacles and its chaotic nature. Group behavior may occur, but has not necessarily to be. Such pedestrian mobility is always limited in speed because the legs act like inverted pendula.

Typical examples of pedestrian mobility in wireless networks are people in the street or mall using cellular phones or PDAs (personal digital assistants), and animals in herds with sensor nodes being observed by biologists, e.g zebras [56]. Upcoming examples of wireless mobility patterns are mobile devices attached to any moving object (parasitic mobility) [31, or even radio devices for pets. A side effect is that pedestrian clients have limited energy-resources. So they need to carry their batteries around to communicate, by that imposing further restraints to the communication network.

\subsection{Marine and Submarine Mobility}

Like pedestrians boats and vessels are limited by an intrinsic maximum speed, resulting from the friction in the water and the available motor power. Unlike in vehicular (earth bound) mobility the motion is truly two-dimensional, and in the case of submarine mobility even three-dimensional, whereas for most cases no group mobility is involved (except regattas, fleet operation and fish swarms). The communication upon the water service is very good and only the globe's curvature may become a problem.

Water absorbs radio signals with high frequencies. Submarine boats circumvent this problem by using very low frequencies ranging form $15 \mathrm{kHz}$ to $33 \mathrm{kHz}$ with an antenna length of 10 to 20 meters. A solution to this problem is acoustic communication, since sound travels very well underwater 2. Also under water speed is the decisive limitation to mobility. Unlike in aerial mobility ascent and descent is easily possible (if the devices are equipped to withstand to the enormous changes of water pressure), so truly three-dimensional movement is realistic.

\subsection{Earth Bound Vehicles}

Such as pedestrian mobility is connected to the pendulum, the vehicular mobility is connected to the wheel. By this term we describe the mobility patterns of cars, railways, bicycles, motor bikes, etc. The wheel based movement reduces friction and allows high speed. So, the danger of collision increases dramatically, and this is the reason why (nearly) all vehicles are bound to one-dimensional movement on streets, paths, or tracks. This reduces the problem of preventing collisions to certain places like crossings. 
For railway traffic there is fixed predictable train schedule and only delays cause aberrations. However, even in the seemingly well-planned world of the railway companies, freight wagons do disappear. This happens very often in the large wide-spread network of railway system spanning over different countries and railway network providers. Therefore, some companies start to use GPSbased wireless tracking devices to locate the wagons.

Another feature of railway traffic is an extreme group mobility pattern. When the passengers are inside a train, then their relative speed reduces to nearly zero, while the whole train can move up to some $300 \mathrm{~km} / \mathrm{h}$. The high relative speed between trains makes direct communication very challenging, especially since they move through a landscape with obstacles, or even tunnels. At these speeds the Doppler-effect starts to kick in. Further the noise of reflections of the scenery decreases the quality of the connection.

A very interesting study of this effect in practice can be seen in [38. It shows the difficulty that already arises if cars traveling on the same road in the same direction communicate with each other over W-LAN, both in simulation and in practice. Even a street sign impacts the quality of communication by its reflection of radio signals.

\subsection{Aerial Mobility}

In this context monitoring flying patterns of migratory birds is a challenging task for biologists. In former times, marking caught exemplars was the only reliable source of information. Nowadays, some birds are equipped with radio tracking devices and can be publicly monitored over the Internet, likewise the monitoring of the black storks by the WWF1.

Flying objects reach high speeds and travel over long distances. Actually birds and airplanes behave quite similar, here. As a communication medium open air is nearly optimal. Still, the signal fades quadratically with the distance, such that multihop communications may reduce the energy consumption of the radio transceiver.

The aerial mobility pattern can be best described as a two and a half dimensional individual movement with limited (yet high) speed. The motion is not completely three-dimensional since each ascent is very costly to the flying object. So, flying objects usually preserve their flight heights. One exception may be air fights between a raptor and his prey, or flight combats between warplanes. Another exception is the group mobility of bird swarms which perfectly coordinate their flight behavior.

The main applications of radio communication in aerial mobility are anticollision systems, message passing, position tracking, and of course flight control.

\subsection{Medium Based Mobility}

One method to explore the interior of hurricanes are dropwindsondes 18. These are sensor devices dropped from an airplane equipped with a parachute, sensors,

\footnotetext{
${ }^{1}$ See http://www.flyingover.net/
} 
GPS-system, and a radio unit. Besides the constant drop speed the main movement comes from the interior winds of the hurricanes. Other wireless devices with medium based mobility are weather balloons and drifting buoys in the oceans. The main application of all these devices is to measure the currents of the medium. Dropwindsondes communicate directly to the aircraft releasing them. Also for other such sensor nodes cellular networks is the prevalent technique to collect the tracking information. A different approach has been used for measuring the currents at the British coast. In [47 a mobile ad hoc network has been used to collect the information of the sensor network over a multihop path (with hardware equipped as few as 384 Bytes of RAM). Using flying sensor devices for the exploration of mars was recently suggested by $[3]$.

To understand the mobility of the sensor devices in the medium one has to study the medium itself. This can be done by numerical solution of the underlying Navier-Stokes-equations. Medium based mobility can be one-, two- or threedimensional depending on the medium and the circumstances (piped gas, surface of fluids, open air). Group behavior can occur and is usually unwanted since grouping sensors deliver less information than individual moving sensors.

\subsection{Mobility in Outer Space}

For radio communication outer space is the perfect environment. Energy for communication is usually no tight resource since space vehicles are equipped with solar paddles. Mobility is, however, restricted since common space ships use rockets for acceleration and fuel supply is limited. Hence, space vehicles drift through space most of the time to save on this resource. At the moment numerous satellites surround the earth forming a mobility pattern of a giant chaotic carrousel, see Fig. 3. But space explorations may produces even more complicated mobility patterns.

Space ships closely fly by planets to increase speed, e.g. Voyager 2 used Saturn's strong gravity to change its course and gather speed (to continue its mission to Uranus and Neptune). Herds of space vehicles may be used for a coordinated view into deep space. These herds will be placed in non-circular orbits between earth and sun. Note that there exist five further stable positions, called Lagrange or libration points (see Fig. 4), between every pair of massive bodies such the sun and its planets, the planets and their moons, and so on. And around these Lagrange points non-circular orbits exist, see Fig 5 . These herds of space vehicles have to change formation from time to time forming complicated mobility patterns. Ad hoc networks will coordinate this movement, prevent collisions and recalibrate the relative positions [29].

\subsection{Robot Motion}

Any of the above mobility scenarios can occur in robot motion. The main difference is the mobility pattern given by the designer of the robots. For some application this pattern is easy to predict, likewise other robots seem to move completely erratic and unpredictable. Clearly, the robot motion is driven by the 


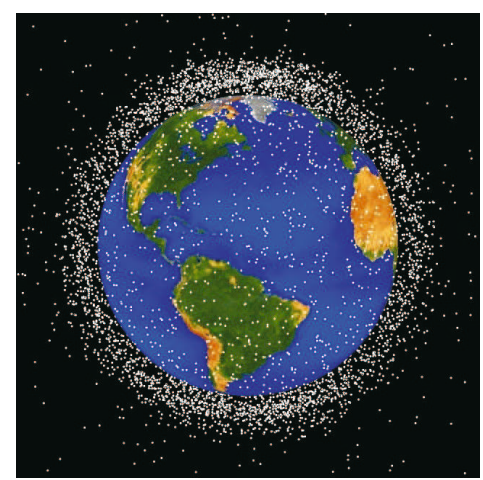

Fig. 3. Space vehicles and debris in low orbit (from NASA Orbital Debris Program Office)

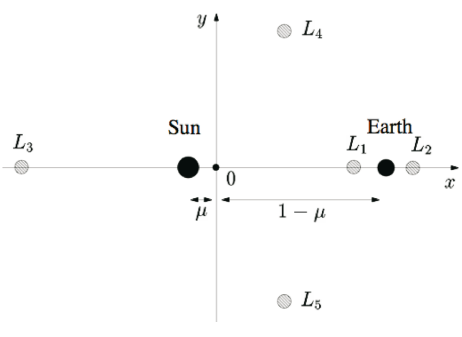

Fig. 4. The five Langrange points [2 28$]$

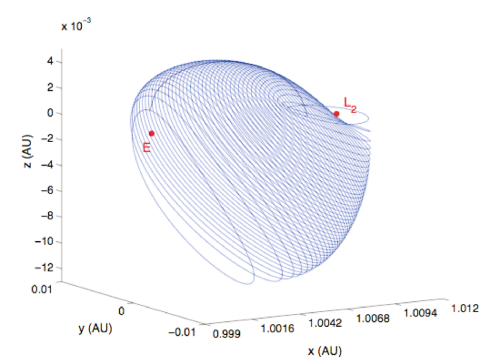

Fig. 5. Family of Halo orbits in the vicinity of the Lagrange point $[2 \$]$

robot's task and usually little attention is given to the impact of the robot's behavior on the communication network.

Currently, in Paderborn the project "Smart Team" make a difference. The goal of this project is to coordinate the robots' task with the necessities of a radio communication network.

\subsection{Characterization of Mobility Patterns}

We have seen that in our modern world mobility is manifold and ubiquitous. Radio communication networks are strongly affected by the different types of mobility and the understanding of each observed mobility pattern can help to improve the network behavior. Throughout this section the following properties played a role.

- Group behavior: Is there a set of nodes staying together for a considerably long time? Clearly, exploiting group behavior improves the performance of radio communication by clustering.

- Limitations: What are the speed and/or acceleration bounds in the mobility pattern?

- Dimensions: Do the nodes move in three-dimensions or only planar or linear?

\footnotetext{
${ }^{2}$ Funded by the DFG SPP 1183 Organic computing.
} 
- Predictability: How well can the behavior of the nodes be predicted, e.g. by a simulation model? Is the behavior completely erratic? Or can it be described by a random process or even by deterministic selfish behavior?

Also hybrid models might appear. In this context we would like to mention the parasitic mobility pattern 31. They present a sensor network where the nodes harvest the mobility from people, animals, vehicles, fluids, cellular organisms, and nature. Furthermore, the nodes can change hosts. Depending on the type of host, parasitic mobility can reproduce nearly all of the above mobility patterns. Such parasitic mobility pattern does not constitute a mobility pattern of its own.

\subsection{Measuring Mobility Patterns by Localization}

To measure a mobility pattern one needs to track a large number of nodes for a long period of time. In fact this is the perfect application area for wireless sensor networks specialized in localization. Localization can be solved by measuring ranging information from signal strength [5], time of arrival [53], or the time difference of arrival 50, or angle of arrival [42. Other localization schemes make use of the quantities of base stations seeding sensors. Some hopcount based techniques avoid this large number of seeding base stations, 43, 45] by relying on a uniform node distribution. In 26] a different approach is presented. They exploit the mobility of the sensor networks to improve the accuracy and reliability of the sensor node localization even if the sensors are seeded by mobile base stations. This is only one of many examples where mobility helps to improve the network behavior.

\section{Models of Mobility}

We classify the mobility models as cellular mobility models, random trip models, group mobility models, particle based models, non-recurrent models, and worst case mobility models.

\subsection{Cellular Mobility Model}

Since for cellular networks the main aspect of mobility is the handoff between cells, one is not particularly interested in every detail of the movement of a mobile node. For a survey for cellular models see 32 .

\section{The Random Walk Model}

In this model, a node stays in a cell or moves to a neighbored cell according to some given transition probabilities. These probabilities are often adjusted to practical observations of client behavior in cells. The Random Walk Mobility Model is one of the most widely used mobility models because it describes individual movements relative to cells $[7,49,58$. Since this model is memoryless, there is no such concept as a path or consecutive movement. Therefore, nodes stay in a vicinity of the starting cell for a rather long time. 
2. Trace based Models

Cellular phone companies have large records of mobility patterns of their users. These traces are a valuable source for the evaluation and improvement of handoff protocols. The only drawback is that usually such data is not publicly available and therefore cannot serve as benchmarks for the scientific community.

3. Fluid Flow Mobility Model

In this model the individual nodes are modeled on a macroscopic level [32, [54, [36], 52]. The behavior of the generated traffic is similar to a fluid flowing through a pipe. As a result, the Fluid-Flow Mobility Model represents traffic on highways very well (for cellular networks). In 33 this model is used to represent the behavioral characteristics of traffic on a highway. [34] shows that the Fluid-Flow Mobility Model is insufficient for individual movements including stopping and starting.

\subsection{Random Trip Mobility}

These mobility models are the prevalent mobility model for MANETs. There are numerous variants of this model. In [12] these models are presented as follows.

1. Random Walk Mobility Model

Each node moves from its current location to a new location by randomly choosing an arbitrary direction and speed from a given range. Such a move is performed either for a constant time for a constant distance traveled. Then new speed and direction are chosen. At the boundaries nodes bounce off like billiard balls on a pool table.

In [34 the Random Walk Mobility Model is described as a memoryless mobility pattern because it retains no knowledge concerning its past locations and speed values. This characteristic inhibits the practicality of the Random Walk Mobility Model because mobile nodes typically have a pre-defined destination and speed in mind, which in turn affects future destinations and speeds.

One observation is that the stationary probability distribution can be described depending on the probabilites. But, the convergence against this stable distribution can be slow, if the points are not randomly chosen [55]. So, there is some danger that the simulation result highly depends on the start position, if the simulation time is not long enough.

In the Smooth Random Mobility Model [9] an extension of the simpler random walk model is given. Here, two independent stochastic processes choose direction and speed changes. The new speeds (or directions) are chosen from a weighted distribution of preferred speeds. Upon a trigger, the speed (resp. direction) changes as determined by a Poisson process.

2. Random Waypoint Mobility Model

The model is equivalent to the Random Walk model except that before any change of speed and direction a predetermined pause time is performed [11]. This model is widely used for evaluating ad hoc network routing protocols. 
3. Random Direction Mobility Model

Here, the node must travel to the edge of the simulation area (or some other condition is met) at a constant speed and direction. Then, the nodes pause and a new direction and velocity is chosen randomly [48. Then, the process repeats.

4. A Boundless Simulation Area Mobility Model: The model exchanges the planar rectangular simulation field by a boundless torus.

5. Gauss-Markov Mobility Model: A model that uses one tuning parameter to vary the degree of randomness in the mobility pattern.

The Random Gauss-Markov Mobility Model is introduced as an improvement over the Smooth Random mobility model 34]. A node's next location is generated by its past location and velocity. Depending upon parameters set, this allows modeling along a spectrum from Random Walk to Fluid-Flow.

6. A Probabilistic Version of the Random Walk Mobility Model [15] In this model the last step made by the random walk influences the next one. Under the condition that a node has moved to the right the probability that it continues to move in this direction is then higher than to stop movement. This leads to a walk that leaves the starting point much faster than the original random walk model.

7. City Section Mobility Model [17]

Here the random waypoint movement is combined with a street map of a virtual city. The paths of the mobile nodes are limited to these streets in the field. In a related model, the streets are replaced by Voronoi graphs [27. Furthermore, obstacle are used which obstruct also radio signals.

For some models there is a slow convergence towards the stationary distribution [40. This influences simulation results, since in previous work simulation usually starts with the uniform distribution which is not necessarily the stationary distribution of the mobility model. Some random waypoint models do not provide a stationary distribution at all. These problems are mentioned in 55] for many random waypoint mobility models.

In [10] the Random Trip model has ben defined. This model describes a wide class of mobility models, contain most of the mobility models in this section. Therefore we use this name for this class of mobility models. Examples include random waypoint on general connected domain, restricted random waypoint, random walk models with either wrap-around or reflection, and the city street model. In [10] it is shown how a simulation algorithm can start from unique steady-state distribution. So, no time must be spent for waiting until the random process stabilizes in the simulator.

\subsection{Group-Mobility Models}

The group-mobility models are usually an extension of the above models, where either a function describes the group behavior or the nodes are somehow associated with a group leader or a target. For a more extensive description of these models we refer to [12] and [25]. 
1. Exponential Correlated Random Mobility Model: Here a motion function creates a group behavior.

2. Column Mobility Model: The set of mobile nodes form a line and move forward in a particular direction.

3. Nomadic Community Mobility Model: A group mobility model where a set of MNs move together from one location to another.

4. Pursue Mobility Model: For each group the group members follow a target node moving over the simulation area.

5. Reference Point Group Mobility Model: The group movement is based upon the path traveled by a logical center. Again the logical center moves according to an individual mobility model.

\subsection{Particle Based Mobility}

There has been a lot of research in predicting pedestrian behavior. One of the main motivations is to understand erratic mass panic caused by many pedestrians causing the death and injuries of hundreds of people in a single event 24. The best model to describe the individual behavior of each person in such occasions is a particle based model 23. Each person is characterized by a sum of forces, describing his desire to move in a direction, keeping a distance to others and the result of contact and frictions with other persons. The simplicity and the accuracy of this model is surprising. It allows even to simulate typical behavior in crowded streets where strangers form queue patterns.

\subsection{Combined Mobility Models}

Many the above mobility models have been combined in a number of theoretical frameworks, simulation environments and analysis toolboxes [6], [9], 22.

\subsection{Non-recurrent Models}

In the context of computational geometry Basch et al. introduced the concept of kinetic data structures (KDS) [8] which describes a framework for analyzing algorithms on mobile objects. In this model the mobility of objects is described by pseudo-algebraic functions of time. These functions are fully or partially predictable. The analysis of a KDS is done by counting the combinatorial changes of the geometric structure that is maintained by the KDS. The worst case mobility depends, therefore, on the specific application for which the KDS is designed. Another approach capturing unpredictable mobility is the concept of soft kinetic data structures (SKDS) [16].

Usually the underlying trajectories of the points are described by polynomials. Then, the corresponding Davenport-Schinzel-sequences [1] can be used to receive an overall bound on the number of events. Because of the polynomials these trajectory eventually move to infinity, which is somehow the worst case for a wireless network. 
The idea of kinetic data structures is also used in [19] to maintain a clustering of moving objects. This approach is used in 20. to determine the head of each cluster in a mobile network. In each cluster the nodes are directly connected to the head. To react on mobility the clustering is updated by an event-based kinetic data structure.

Another non-recurrent approach has been proposed in [37]. They investigate a contraction mobility model, an expansion mobility model, and a circling mobility model (which is the only recurrent model). In the contraction model the nodes move toward a center on a straight line. Within some time interval a new speed will be chose from time interval and in addition the nodes may pause. The expansion model is the same model, but now the nodes move from the center on some beams. In the circling model the nodes move on concentric circles around a center. These mobility models can be combined with a street network.

\subsection{Worst-Case Mobility Models}

A worst case model is introduced in 51. Here, any movement is allowed as long as it is bounded by a velocity or an acceleration bound. The authors call the first model the pedestrian mobility model. Here all mobile nodes obey a system-wide speed limit. The other model, where all mobile nodes can move arbitrarily fast, yet obey the same acceleration bound, is called vehicular mobility. Based on this worst case assumption the authors try to maintain a network for some constant amount of time and then allow to completely rebuild the infrastructure. For this the location (and speed vector) at the beginning of a round is known, yet further movement is completely unpredictable (within the limits). So, the transmission length needs to be adjusted appropriately. In this model the authors investigate the quality of the topology control similar to the models presented in [4].

In this worst-case approach scenarios may appear where all networks have bad performance. These scenarios are caused by large crowds of mobile nodes. They introduce a location dependent measure, called crowdedness, and can prove for restricted crowdedness that the optimal network topology can be approximated in both mobility models by the so-called Hierarchical Grid topology.

\section{Discussion}

\subsection{Mobility is Helpful}

One might think that mobility has only a negative impact on the behavior of wireless networks. But recent work has shown that this is not the case. Mobility improves the coverage of wireless sensor networks [35. It helps security in ad hoc networks [13. Furthermore it can help with network congestion as shown in 21. This approach overcomes the natural lower bound for throughput of $\Omega(\sqrt{n})$ by instrumenting the random movement of nodes. They design a protocol where mobile nodes relay packets and literally transport them towards the destination node. 


\subsection{Mobility Models and Reality}

There is an obvious discrepancy between the manifold mobility pattern observable in reality and the mobility models used as benchmark tools and as theoretical models for wireless networks. The prevalent mobility are the random trip models. It relies on the assumption that individuals move more or less erratically. Some of the random trip models have been adapted with realistic assumptions like street maps, velocity bounds, etc. Yet, on the one hand it is still unproven whether these modifications describe realistic mobility patterns. And even if this is the case they describe only the earthbound pedestrian or vehicular mobility patterns.

In the case of group mobility, little information is available on how real group mobility patterns look like. Sometimes group mobility patterns are not caused by social interaction but by a physical process. As an example, pedestrians in crowded pathways form queues merely to avoid the approaching pedestrians [23]. At the moment little is known whether the group mobility models actually describe the reality.

The worst-case mobility approach seems to be a step towards a more general understanding of mobility. Some drawbacks need to be mentioned. First, it relies on homogeneous velocity or acceleration bounds, which is not at all realistic. Second, the implications for wireless networks are rather weak. For that, the performance of the network depends very much of the crowdedness of the underlying mobility pattern.

In principle, is possible to formulate the missing mobility models for marine, aerial, medium based, and outer space mobility patterns. Also for the pedestrian and vehicular models we expect even more realistic mobility models to be considered as benchmarks for wireless networks. The research of mobility models is quite vivid. Nevertheless, some challenges remain:

- Find mobility models for specific mobility patterns and prove their validity by comparing them with reality.

- Prove the efficiency and reliability of a real network protocols with respect to a given mobility model.

\section{References}

1. Agarwal, P.K., and Sharir, M.: Davenport-Schinzel Sequences and Their Geometric Applications. Cambridge, New York, Cambridge University Press (1995)

2. Akyildiz, I.F., Pompili, D., and Melodia, T.: Underwater Acoustic Sensor Networks: Research Challenges. Ad Hoc Networks (Elsevier) 33 (May 2005) 257-279

3. Antol, J., Calhoun, P., Flick, J., Hajos, G.A., Kolacinski, R., Minton, D., Owens, R., and Parker, J.: Low Cost Mars Surface Exploration: The Mars Tumbleweed. Technical Report, NASA Langley Research Center. NASA/TM 2003212411 (August 2003)

4. Meyer auf der Heide, F., Schindelhauer, C., Volbert, K., and Grünewald, M.: Energy, Congestion, and Dilation in Radio Networks. In Proceedings of the 14th Annual ACM Symposium on Parallel ALgorithms and Architectures (SPAA-02), New York, ACM Press, August 10-13 2002, 230-237 
5. Bahl, P., and Padmanabhan, V.N.: RADAR: An in-Building RF-Based User Location and Tracking System. In INFOCOM 2 (2000) 775-784

6. Bai, F., Sadagopan, N., and Helmy, A.: Important: a Framework to Systematically Analyze the Impact of Mobility on Performance of Routing Protocols for Adhoc Networks. In Proceedings of INFOCOM 2003, San Francisco, CA. (2003)

7. Bar-Noy, A., Kessler, I., and Sidi, M.: Mobile Users: To Update or Not to Update. In IEEE Conference on Computer Communications (INFOCOM 94) (1994) 570576

8. Basch, J., Guibas, L.J., and Hershberger, J.: Data Structures for Mobile Data. Journal of Algorithms 311 (1999) 1-28

9. Bettstetter, C.: Smooth is Better than Sharp: a Random Mobility Model for Simulation of Wireless Networks. In MSWIM '01: Proceedings of the 4th ACM International Workshop on Modeling, Analysis and Simulation of Wireless and Mobile Systems, ACM Press (2001) 19-27

10. Le Boudec, J., and Vojnovic, M.: Perfect Simulation and Stationarity of a Class of Mobility Models. In Proceedings of IEEE INFOCOM '05 (2005), to appear in

11. Broch, J., Maltz, D.A., Johnson, D.B., Hu, Y.-C., and Jetcheva, J.: A Performance Comparison of Multi-Hop Wireless ad Hoc Network Routing Protocols. In Mobile Computing and Networking (1998) 85-97

12. Camp, T., Boleng, J., and Davies, V.: A Survey of Mobility Models for Ad Hoc Network Research. Wireless Communications \& Mobile Computing (WCMC): Special issue on Mobile Ad Hoc Networking: Research, Trends and Applications, 25 (2002) 483-502

13. Capkun, S., Hubaux, J.-P., and Buttyán, L.: Mobility Helps Security in Ad Hoc Networks. In MobiHoc '03: Proceedings of the 4th ACM International Symposium on Mobile Ad Hoc Networking \& Computing, ACM Press, (2003) 46-56

14. Chatzigiannakis, I., Dimitriou, T., Mavronicolas, M., Nikoletseas, S., and Spirakis, P.: A Comparative Study of Protocols for Efficient Data Propagation in Smart Dust Networks. In Lecture Notes in Computer Science 2790 (2003) 10031016

15. Chiang, C.: Wireless Network Multicasting. PhD thesis, University of California, Los Angeles (1998)

16. Czumaj, A., and Sohler, C.: Soft Kinetic Data Structures. In Symposium on Discrete Algorithms (SODA’01) (2001) 865-872

17. Davies, V.: Evaluating Mobility Models within an Ad Hoc Network. Master's Thesis, Colorado School of Mines (2000)

18. Franklin, J.L., Black, M.L., and Valde, K.: Eyewall Wind Profiles in Hurricanes Determined by gps Dropwindsondes. Technical Report, NOAA/ National Weather Service National Centers for Environmental Prediction National Hurricane Center (2000)

19. Gao, J., Guibas, L.J., Hershberger, J., Zhang, L., and Zhu, A.: Discrete Mobile Centers. In Proc. of the 17th Symposium on Computational Geometry (SOCG'01) (2001) 188-196

20. Gao, J., Guibas, L.J., Hershberger, J., Zhang, L., and Zhu A.: Geometric Spanner for Routing in Mobile Networks. In ACM Symposium on Mobile Ad Hoc Networking and Computing (MOBICOM'01) (2001) 45-55

21. Grossglauser, M., and Tse, D.N.C.: Mobility Increases the Capacity of Ad Hoc Wireless Networks. IEEE/ACM Trans. Netw. 104 (2002) 477-486

22. Haerri, J., Filali, F., and Bonnet, C.: A Framework for Mobility Models Generation and Its Application to Inter-Vehicular Networks. In MobiWac 2005, The 3rd IEEE 
International Workshop on Mobility Management and Wireless Access, June 13-16, 2005, Maui, Hawaii, U.S.A. (2005)

23. Helbing, D., Farkas, I., Molnar, P., and Vicsek, T.: Simulation of Pedestrian Crowds in Normal and Evacuation Situations. In M. Schreckenberg and S.D. Sharma (eds.) Pedestrian and Evacuation Dynamics (Springer, Berlin) (2002) 21-58

24. Helbing, D., Farkas, I., and Vicsek, T.: Simulating Dynamical Features of Escape Panic. Nature 407 (2000) 487-490

25. Hong, X., Gerla, M., Pei, G., and Chiang, C.-C.: A Group Mobility Model for Ad Hoc Wireless Networks. In Proc. of the 2nd ACM int. workshop on Modeling, Analysis and Simulation of Wireless and Mobile Systems (1999) 53-60

26. Hu, L., and Evans, D.: Localization for Mobile Sensor Networks. In MobiCom '04: Proceedings of the 10th Annual International Conference on Mobile Computing and Networking, ACM Press, (2004) 45-57

27. Jardosh, A., Belding-Royer, E.M., Almeroth, K.C., and Suri, S.: Towards Realistic Mobility Models for Mobile Ad Hoc Networks. In MobiCom '03: Proceedings of the 9th Annual International Conference on Mobile Computing and Networking, ACM Press (2003) 217-229

28. Junge, O., Levenhagen, J., Seifried, A., and Dellnitz, M.: Identification of Halo Orbits for Energy Efficient Formation Flying. In Proceedings of the International Symposium Formation Flying, Toulouse (2002)

29. Junge, O., and Ober-Blöbaum, S.: Optimal Reconfiguration of Formation Flying Satellites. In Accepted for IEEE Conference on Decision and Control and European Control Conference ECC 2005, Seville, Spain (2005)

30. Karl, H., and Willig, A.: Protocols and Architectures for Wireless Sensor Networks. John Wiley \& Sons (2005)

31. Laibowitz, M., and Paradiso, J.A.: Parasitic Mobility for Pervasive Sensor Networks. In H.W. Gellersen, R. Want and A. Schmidt (eds), Pervasive Computing, Third International Conference, PERVASIVE 2005, Munich, Germany, May 2005, Proceedings. Springer-Verlag, Berlin (2005)s 255-278

32. Lam, D., Cox, D., and Widom, J.: Teletraffic Modeling for Personal Communications Services. IEEE Communications Magazine Special Issue on Teletraffic Modeling, Engineering and Management in Wireless and Broadband Networks 35 2 (February 1997) 79-87

33. Leung, K.K., Massey, W.A., and Whitt, W.: Traffic Models for Wireless Communication Networks. In INFOCOM 3 (1994) 1029-1037

34. Liang, B., and Haas, Z.J.: Predictive Distance-Based Mobility Management for PCS Networks. In Proceedings of IEEE INFOCOM'99 (1999) 1377-1384

35. Liu, B., Brass, P., Dousse, O., Nain, P., and Towsley, D.: Mobility Improves Coverage of Sensor Networks. In MobiHoc '05: Proceedings of the 6th ACM International Symposium on Mobile Ad Hoc Networking and Computing, New York, NY, USA, ACM Press (2005) 300-308

36. Lo, C.N., Wolff, R.S., and Bernhardt, R.C.: An Estimate of Network Database Transaction Volume to Support Universal Personal Communication Services. In 1st Int. Conf. Universal Personal Communications (1992) 09.03/16

37. Lu, Y., Lin, H., Gu, Y., and Helmy, A.: Towards Mobility-Rich Performance Analysis of Routing Protocols in Ad Hoc Networks: Using Contraction, Expansion and Hybrid Models. In IEEE International Conference on Communications (ICC) (June 2004)

38. Maurer, J.: Strahlenoptisches Kanalmodell für die Fahrzeug-Fahrzeug-Funkkommunikation. PhD Thesis, Universität Fridericiana Karlsruhe (2005) 
39. Mauve, M., Widmer, J., and Hartenstein, H.: A Survey on Position-Based Routing in Mobile Ad Hoc Networks. IEEE Network Magazine 156 (November 2001) 30-39

40. McGuire, M.: Stationary Distributions of Random Walk Mobility Models for Wireless Ad Hoc Networks. In MobiHoc '05: Proceedings of the 6th ACM International Symposium on Mobile Ad Hoc Networking and Computing, ACM Press (2005) 90-98

41. Nain, P., Towsley, D., Liu, B., and Liu, Z.: Properties of Random Direction Models. Technical Report, INRIA Technical Report RR-5284 (July 1994)

42. Niculescu, D., and Nath, B.: Ad Hoc Positioning System (APS) Using AoA. In Proceedings of INFOCOM 2003, San Francisco, CA. (2003)

43. Niculescu, D., and Nath, B.: Position and Orientation in Ad Hoc Networks. Ad Hoc Networks 2 (2004) 133-151

44. Perkins, C.E.: Ad Hoc Networking. Addision-Wesley (2001)

45. Nagpal, J.B.R., and Shrobe, H.: Organizing a Global Coordinate System from Local Information on an Ad Hoc Sensor Network. Lecture Notes in Computer Science 2634 (January 2003) 333-348

46. Raghavendra, C.S., Sivalingam, K.M., and Znati, T., (eds): Wireless Sensor Networks. Springer (2005)

47. Roadknight, C., Gonzalez, A., Parrot, L., Boult, S., and Marshall, I.: An Intelligent Sensor Network for Oceanographic Data Acquisition. In AD HOC NOW 2005 4th International Conference on Ad Hoc Networks and Wireless Cancun (October $6-8,2005)$

48. Royer, E.M., Melliar-Smith, P.M., and Moser, L.E.: An Analysis of the Optimum Node Density for Ad Hoc Mobile Networks. ICC 2001 - IEEE International Conference on Communications (June 2001) 857-861

49. Rubin, I., and Choi, C.: Impact of the Location Area Structure on the Performance of Signaling Channels in Wireless Cellular Networks. IEEE Communications Magazine (February 1997) 108-115

50. Savvides, A., Han, C.-C., and Strivastava, M.B.: Dynamic Fine-Grained Localization in Ad-Hoc Networks of Sensors. In Mobile Computing and Networking (2001) 166-179

51. Schindelhauer, C., Lukovszki, T., Rührup, S., and Volbert, K.: Worst Case Mobility in Ad Hoc Networks. In Proceedings of the 15th Annual ACM Symposium on Parallelism in Algorithms and Architectures (SPAA-03), New York, ACM Press (June 7-9 2003) 230-239

52. Frost, B.M.V.: Traffic Modeling for Telecommunications Networks. IEEE Communications Magazine 323 (March 1994) 70-81

53. Wellenhoff, B.H., Lichtenegger, H., and Collins, J.: Global Positioning System: Theory and Practice. Springer Verlag (1997)

54. Xie, H., Tabbane, S., and Goodman, D.: Dynamic Location Area Management and Performance Analysis. In IEEE VTC'93 (1993) 536-539

55. Yoon, J., Liu, M., and Noble, B.: Random Waypoint Considered Harmful. In Proceedings of INFOCOM. IEEE (2003)

56. Zhang, P., Sadler, C.M., Lyon, S.A., and Martonosi, M.: Hardware Design Experiences in Zebranet. In SenSys '04: Proceedings of the 2nd International Conference on Embedded Networked Sensor Systems, ACM Press (2004) 227-238

57. Zhao, F., and Guibas, L.: Wireless Sensor Networks: An Information Processing Approach. Morgan Kaufmann (2004)

58. Zonoozi, M., and Dassanayake, P.: User Mobility Modeling and Characterization of Mobility Pattern. IEEE Journal on Selected Areas in Communications 151 (September 1997) 1239-1252 